\title{
Size-Dependent Indirect Excitation of Trivalent Er Ions via Si Nanocrystals Embedded in a Silicon-Rich Silicon Oxide Matrix Deposited by ECR-PECVD
}

\author{
G. Zatryb, ${ }^{1}$ A. Podhorodecki, ${ }^{1}$ J. Misiewicz, ${ }^{1}{ }^{\text {J. }}$ Wojcik, ${ }^{2}$ and P. Mascher ${ }^{2}$ \\ ${ }^{1}$ Institute of Physics, Wroclaw University of Technology, Wybrzeze Wyspianskiego 27, 50-370 Wroclaw, Poland \\ ${ }^{2}$ Centre for Emerging Device Technologies, Hamilton, ON, Canada L8S 4L7 \\ Correspondence should be addressed to G. Zatryb, grzegorz.zatryb@pwr.wroc.pl
}

Received 21 July 2009; Revised 7 October 2009; Accepted 23 December 2009

Recommended by John A. Capobianco

Silicon nanocrystals ( $\mathrm{Si}-\mathrm{nc}$ ) embedded in a silicon-rich silicon oxide matrix codoped with $\mathrm{Er}^{3+}$ ions have been fabricated by electron-cyclotron plasma-enhanced chemical vapor deposition. Indirect excitation of erbium photoluminescence via silicon nanocrystals has been obtained within a broad pump wavelength range. The influence of different nanocrystal sizes on the excitation transfer from the $\mathrm{Si}-\mathrm{nc}$ to $\mathrm{Er}^{3+}$ ions is discussed.

Copyright ( $) 2009$ G. Zatryb et al. This is an open access article distributed under the Creative Commons Attribution License, which permits unrestricted use, distribution, and reproduction in any medium, provided the original work is properly cited.

\section{Introduction}

Erbium codoped silicon nanocrystals have become a subject of significant interest due to the wavelength of the radiative transition ${ }^{4} I_{13 / 2} \rightarrow{ }^{4} I_{15 / 2}$ in the $4 \mathrm{f}$ shell of the $\mathrm{Er}^{3+}$ ion $(1.54 \mu \mathrm{m})$ which lies in the spectral region of minimum dispersion and maximum transparency of quartz opticalfiber telecommunication lines. Indirect excitation of erbium ions through electronic states of the silicon oxide host is known to be much more efficient than resonant optical pumping of the $4 \mathrm{f}$ electron shell of erbium ion itself [1]. The sensitization process allows one to increase the effective absorption cross-section of erbium by about three orders of magnitude [2]. Additionally, in the presence of silicon nanocrystals ( $\mathrm{Si}-\mathrm{nc}$ ) an absorption band covers the wide visible range and thus lanthanide ions can be excited indirectly by white light.

Several mechanisms have been proposed to explain the sensitization process of $\mathrm{Er}^{3+} \mathrm{PL}$ in systems containing $\mathrm{Si}$ nc. It has been proposed that mainly two energy transfer processes takes place, whose occurrence depends on the ion position with respect to Si-nc and which can work simultaneously [3]. The first process, for the ions very close to the Si-nc surface is fast with transfer time less than $100 \mathrm{~ns}$. In this process an exciton is trapped to a defect state in the band gap and transfers its recombination energy to $\mathrm{Er}^{3+}$ ion by Auger-like process. The second energy transfer process is slower and is considered to be due to the resonant Förster type Coulombic interaction between the free excitons in Si-nc and $\mathrm{Er}^{3+}$. This kind of transfer can occur between nanocrystals and ions that are located at some distance from one another.

Another factor that play a role in case of sensitization process is electronic structure of Si-nc. Several features are important here, like for example the spacing of energy levels of Si-nc, tuning of Si-nc and $\mathrm{Er}^{3+}$ electronic states from which energy transfer occurs [4], existence of some interface states like $\mathrm{Si}=\mathrm{O}[5]$ and passivation of $\mathrm{Si}-\mathrm{nc}$ surface for instance by nitrogen [6]. Even though there has been much research activity in this field, details of the influence of Si-nc electronic structure on the excitation of $\mathrm{Er}^{3+}$ ions are not yet sufficiently well understood. In particular, there is a shortage of excitation data in the high-absorption spectral region of Si-nc. For instance, available photoluminescence excitation (PLE) results obtained for Si-nc: $\mathrm{Er}^{3+}$ systems focus rather on low-energetic part of the PLE spectrum [7] or does not reach sufficiently deep [8] in the ultra-violet (UV) range where the absorption of Si-nc is very strong. Nevertheless, this kind of results are very valuable since they allowed to establish the occurrence of mediated excitation in this kind of systems. 
However, it is frequently not enough if one would like to investigate the influence of Si-nc absorption properties on excitation of the $\mathrm{Er}^{3+}$ ions.

In this paper, we present results of an investigation of the excitation of erbium photoluminescence in a broad spectral range and its dependence on the erbium concentration and on different silicon concentrations in silicon-rich silicon oxide (SRSO) films grown by electron-cyclotron resonance plasma enhanced chemical vapor deposition (ECR)-PECVD.

\section{Experimental Details}

The Er-codoped SRSO thin films were grown on single crystal Si substrates by ECR-PECVD using $\mathrm{SiH}_{4}$ and $\mathrm{O}_{2}$ source gases diluted in Ar to form the SRSO matrix. $\operatorname{Er}(\mathrm{TMHD})_{3}$ (where TMHD stands for 2,2,6,6-tetramethyl3,5-heptanedionato) was employed as the rare-earth precursor to achieve high-concentrations of Er codoping. The rare-earth sources were contained in a manifold which can be heated up to $200^{\circ} \mathrm{C}$ to sublime the precursor inside. Ar carrier gas then transports the rare-earth precursor through a transmission line after which it is introduced in the chamber alongside the $\mathrm{SiH}_{4}$ through the lower dispersion ring. All the films were annealed in a quartz tube furnace under flowing ultrahigh purity $\mathrm{N}_{2}$ for 1 hour. The annealing temperature was in all cases $1100^{\circ} \mathrm{C}$. As we have shown in many papers in case of our deposition system this temperature is sufficient to obtain a few nanometers large silicon nanocrystals. This effect has been shown in the absence of erbium codoping [9] as well as for erbium and different lanthanides codoped samples $[10,11]$. The deposition system has been described in more details elsewhere [11]. The composition of the films was measured by Rutherford Backscattering Spectroscopy.

The room temperature photoluminescence (PL) and photoluminescence excitation (PLE) of the erbium ions were measured using an InGaAs pin photodiode. As an excitation source, a $450 \mathrm{~W}$ Xe arc lamp connected to Triax 180 Jobin Yvon monochromator was used.

\section{Results and Discussion}

Figure 1 shows PL spectra in the infrared region obtained for $\mathrm{Er}^{3+}$ codoped $\mathrm{Si}_{x} \mathrm{O}_{y}$ films at $488 \mathrm{~nm}$ excitation wavelength. The Si concentration in the films was 37 and 39 at. \%, with $\mathrm{Er}^{3+}$ dopant concentrations of $0.15,0.30$ and 0.45 at. \%. For all samples, emission at $1534 \mathrm{~nm}$ was observed, which is related to ${ }^{4} I_{13 / 2} \rightarrow{ }^{4} I_{15 / 2}$ transition of $\mathrm{Er}^{3+}$ ions. The complex structure of the emission band likely originates from different environments of the $\mathrm{Er}^{3+}$ ions. This results in slightly shifted energy levels of the emitting ions, which are differently split by the local crystal field.

Moreover, Figure 1 shows that the PL intensity increases strongly with the increase in erbium concentration and reaches a maximum for 0.45 at. \%. Overall, the strongest emission was obtained for the sample with 39 at. \% Si. The observed changes of the PL intensity with different Si and Er concentrations are consistent with our previous results [12] and are discussed there.
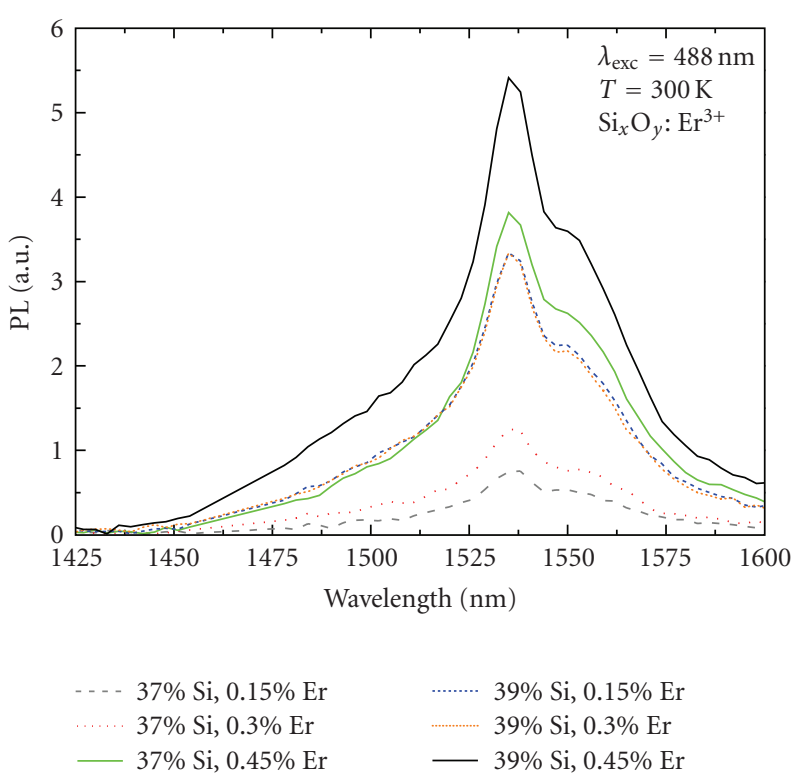

Figure 1: PL spectra of $\mathrm{Er}^{3+}$ codoped $\mathrm{Si}_{x} \mathrm{O}_{y}$ films with different at. $\%$ of Si and Er obtained at $488 \mathrm{~nm}$ excitation wavelength.

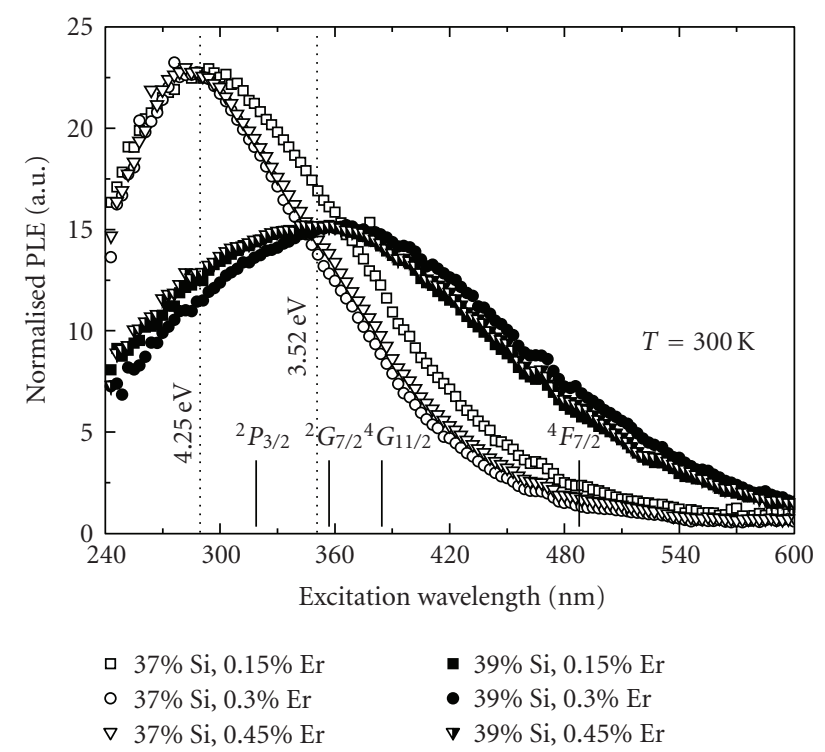

FIgURE 2: Normalized PLE spectra of $\mathrm{Er}^{3+}$ codoped $\mathrm{Si}_{x} \mathrm{O}_{y}$ films with different at. \% of Si and Er measured at $1534 \mathrm{~nm}$.

It is well known that increasing the Si concentration in SRSO results in the formation of larger nanocrystals with smaller band gap $[13,14]$. Thus, by changing the nanocrystal size, it is possible to tune the energy levels and to obtain more efficient excitation of the lanthanide ions either by a resonant or by a nonresonant excitation transfer. To verify this scenario we performed PLE experiments of $\mathrm{Er}^{3+}$ ions, comparing the properties of samples with 37 and 39 at. \% Si, respectively. The obtained results are shown in Figure 2, in the form of normalized PLE intensities. The normalization 
was done separately for samples with 37 and 39 at. \% Si relative to samples with the 0.45 at. \% of erbium ions.

The appearance of $\mathrm{Er}^{3+}$ related emission at excitation wavelengths far from resonance with erbium energy levels clearly indicates an efficient excitation transfer from silicon nanocrystals to lanthanide ions. From Figure 2, it is also evident that the maximum of the PLE peak shifts significantly towards smaller energies when the Si content increases, namely from $4.25 \mathrm{eV}$ (for 37 at. \% Si) to $3.52 \mathrm{eV}$ (for 39 at. \% Si). At the same time any significant shift in the PLE spectrum for different erbium concentrations is observed. The latter can be related to the small erbium concentrations while the former is related to quantum confinement effects (QCE), since changes in Si concentration reflect changes in Si-nc sizes [13].

Moreover, the shape of the PLE spectra for samples with 39 at. \% Si is much more Gaussian than in the case of 37 at. $\%$ Si. From fitting the PLE curves with a Gaussian function, we obtain a FWHM (full width at half maximum) of about $\sim 167 \mathrm{~nm}$ for samples with 37 at. \% Si and $\sim 210 \mathrm{~nm}$ for samples with 39 at. \% Si. It is well known that during the PECVD process the crystals' mean radius increases with the silicon concentration of the films. Larger mean radii are also associated with a wider size distribution of Si nanocrystals. For example, Iacona et al. [13] have demonstrated that for nanocrystals deposited by PECVD with 37 at. \% Si the radius is $r=1.1 \mathrm{~nm}$ with standard deviation $\sigma=0.2 \mathrm{~nm}$, for 39 at. \% $\mathrm{Si}, r=1.5$ and $\sigma=0.3$, and for 42 at. $\% \mathrm{Si}, \mathrm{r}=1.7$ with $\sigma=0.5$. These changes in size distribution should also be reflected in their absorption spectra [15]. Thus, the changes of the PLE spectra observed in our experiment may be associated with a widening of the nanocrystal size distribution and changes of the distribution mean value (QCE) [15].

It should be pointed out that in general, the PLE signal is not simply an absorption spectrum of silicon nor the erbium ions. This is due to the fact that emission from the lanthanide ion depends on the absorption efficiency of Si-nc, the emission efficiency of the erbium ions, but also on the efficiency of the excitation transfer from the nanocrystal to the ion. This last factor can be dependent on the kind of the excitation transfer mechanism that takes place (Förster-Dexter energy transfer or charge transfer) and the matching of the energy levels of the nanocrystal and ion. Assuming constant erbium-related emission efficiency, our experimental results support the notion that the absorption properties of Si nanocrystals themselves play a dominant role in the excitation of $\mathrm{Er}^{3+}$ ions since the excitation spectra of the ions depend on the Si-nc size and size distribution in the same way as it should be expected for the Si-nc alone [16-18]. Unfortunately, the strong excitation transfer weakens the PL related to Si nanocrystals dramatically and we were not able to detect the PLE originating from Si-nc in our case.

It is also worth of noticing, that shift of energy value of the PLE maximum for only a 2 at. \% change in $\mathrm{Si}$ concentration is very strong (around $0.7 \mathrm{eV}$ ). It should not be however surprising when one takes into account that even a small change in Si concentration may lead to a significant change in the Si-nc sizes. As we have shown previously [9], the change of Si-nc diameter is smaller for larger annealing temperatures but is still very well recognizable for $\mathrm{T}_{a}=1100^{\circ} \mathrm{C}$ and for instance 3 at. \% difference in $\mathrm{Si}$ concentration may lead to 1-2 nm change of Si-nc size.

Moreover, these few nanometers changes in Si-nc diameter should be considered as very large if one takes into account that we are dealing with the strong quantum confinement regime, where Si-nc size is comparable to the exciton Bohr radius of bulk Si. In this case even a fractional change of Si-nc size may results in a strong change of the nanocrystal band gap. Wolkin et al. have shown [19] that when the nanocrystal size increases from 1 up to about 1.6 $\mathrm{nm}$ the energy gap can decrease from approximately 4.25 down to $3.1 \mathrm{eV}$, respectively. It should be however pointed out that in our case this strong change should be visible rather in the absorption than emission properties of Si-nc, because as we have shown previously [12] we are dealing with recombination via the trapped excitons. Therefore the quantum confinement effect should manifest itself in the PLE results whereas PL spectra of nanocrystals may not even be affected by the Si-nc size due to surface states related emission.

In the regime of small nanocrystal diameters, taking into account the empirical formula given by Meier et al. [15] the oscillator strength increases strongly when nanocrystal diameters is reduced. Therefore, the Si-nc absorption crosssection is expected to be much higher in the case of 37 at. $\%$ Si samples, what should be reflected in more efficient $\mathrm{Er}^{3+}$ emission. Indeed, taking into account PLE intensities before normalization, among three Er concentrations that were used, we obtained the strongest $\mathrm{Er}^{3+}$ emission intensity for samples containing 37 at. \% Si and 0.45 at. \% Er, about 4 times stronger than in the case of 39 at. \% Si and the same Er content. This result is in agreement with the above given interpretation. On the other hand, the HOMO-LUMO gap of Si-nc and the ${ }^{4} I_{13 / 2}$ level of erbium ions are even less well tuned to resonance in the case of smaller Si-nc, due to QCE. This should decrease the ${ }^{4} I_{13 / 2} \rightarrow{ }^{4} I_{15 / 2}$ emission because multistep relaxation or multiphonon processes are needed to reach the emitting state. Nevertheless, we do not observe weakening of the emission for 37 at. \% samples in comparison to 39 at. \% samples. This result allows us to conclude, that in investigated case the Si-nc absorption cross-section is the most important factor that influences Er emission intensity.

In Figure 2, we also show a few higher $\mathrm{Er}^{3+}$ energy levels, since some of them are in resonance with the excitation wavelengths used during the PLE experiment and a weak resonant excitation may also appear. However, we did not observe any evidence of this excitation mechanism in our case. This is mostly because the concentration of the lanthanide ions is very low in the investigated samples, which, together with a very low excitation cross-section of erbium ions $\left(\sim 10^{-20} \mathrm{~cm}^{2}\right)$, should not give any significant contribution to the absorption. Thus, it can be concluded that the erbium excitation is dominated by the optical properties of the silicon nanocrystals.

To analyze the obtained data in more detail, the correlation between the obtained absorption edge and the size of the Si-nc has been estimated based on the formula given 
by Niquet et al [20]. Assuming the mean diameters of Sinc taken from Iacona et al [13] for 37 at. \% Si and 39 at. \% $\mathrm{Si}$ as 2.2 and $3 \mathrm{~nm}$, respectively, values of $2.28 \mathrm{eV}$ and $1.87 \mathrm{eV}$ were obtained, which correspond to HOMOLUMO gap of silicon nanocrystals. To compare these results with our experimental data, the low energy part of the PLE spectra, corresponding to the lowest absorption transitions, was analyzed assuming that the absorption coefficient in this range is well described by the Tauc formula taken for indirect interband transitions of the bulk materials. The absorption edge values obtained in this way are $\mathrm{E}_{g}=2.1 \mathrm{eV}$ for samples with 37 at. $\% \mathrm{Si}$, and $\mathrm{E}_{g}=1.7 \mathrm{eV}$ for samples with 39 at. $\%$. Any deviation in the calculated values for samples that vary in erbium concentration was within experimental error limit. Obtained band gaps are in good agreement with our theoretical estimations and other published experimental results [21]. However, despite the good agreement with the theoretical predictions it must be pointed out that it is only an estimation due to fact that in the case of purely zero-dimensional structures, k-space does not exist (thus, formally, indirect transitions also do not exist) and the density of states is a delta-like function, strongly different from the Tauc assumption of a square-like function for a bulk material.

\section{Conclusions}

The excitation spectra of Er photoluminescence in ECRPECVD deposited SRSO: Er thin films have been measured. It has been shown that in all investigated samples a strong indirect excitation mechanism of $\mathrm{Er}^{3+}$ ions occurs. Moreover, a significant shift of the PLE spectra has been observed and ascribed to the quantum confinement effect. This feature together with considerable broadening of the PLE spectra for wider Si-nc size distribution indicate that the efficiency of indirect excitation of erbium ions is based mostly on the absorption properties of silicon nanocrystals. Thus, increase of Si-nc related absorption results in more efficient ${ }^{4} \mathrm{I}_{13 / 2} \rightarrow$ ${ }^{4} \mathrm{I}_{15 / 2}$ emission, even though the HOMO-LUMO gap of Si-nc and the ${ }^{4} \mathrm{I}_{13 / 2}$ level of erbium ions are less and less well tuned when decreasing the nanocrystal size so that a multistep relaxation or multiphonon processes are needed to reach the emitting state.

\section{Acknowledgments}

The work was supported by the Polish Ministry of Education and Scientific Research within Grant no. N N515 4108 34. G. Zatryb and A. Podhorodecki acknowledge financial support from fellowship co-financed by European Union within European Social Fund. Moreover, the second author acknowledges financial support from the Foundation for Polish Science. In Canada, this work was supported by the Ontario Research and Development Challenge Fund (ORDCF), under the auspices of the Ontario Photonics Consortium.

\section{References}

[1] F. Priolo, G. Franzò, S. Coffa, and A. Carnera, "Excitation and nonradiative deexcitation processes of $\mathrm{Er}^{3+}$ in crystalline Si," Physical Review B, vol. 57, no. 8, pp. 4443-4455, 1998.

[2] F. Priolo, G. Franzò, D. Pacifici, V. Vinciguerra, F. Iacona, and A. Irrera, "Role of the energy transfer in the optical properties of undoped and Er-doped interacting Si nanocrystals," Journal of Applied Physics, vol. 89, no. 1, pp. 264-272, 2001.

[3] M. Fujii, K. Imakita, K. Watanabe, and S. Hayashi, "Coexistence of two different energy transfer processes in $\mathrm{SiO}_{2}$ films containing Si nanocrystals and Er," Journal of Applied Physics, vol. 95, no. 1, pp. 272-280, 2004.

[4] K. Imakita, M. Fujii, and S. Hayashi, "Spectrally resolved energy transfer from excitons in Si nanocrystals to Er ions," Physical Review B, vol. 71, no. 19, Article ID 193301, 4 pages, 2005.

[5] A. J. Kenyon, M. Wojdak, I. Ahmad, W. H. Loh, and C. J. Oton, "Generalized rate-equation analysis of excitation exchange between silicon nanoclusters and erbium ions," Physical Review B, vol. 77, no. 3, Article ID 035318, 9 pages, 2008.

[6] M. Wojdak, I. I. Liaw, I. Ahmad, et al., "Silicon nanoclusters containing nitrogen and sensitization of erbium luminescence in $\mathrm{SiO}_{x}$ :Er," Materials Science and Engineering B, vol. 146, no. 1-3, pp. 175-178, 2008.

[7] G. Franzò, E. Pecora, F. Priolo, and F. Iacona, "Role of the $\mathrm{Si}$ excess on the excitation of Er doped $\mathrm{SiO}_{x}$," Applied Physics Letters, vol. 90, no. 18, Article ID 183102, 2007.

[8] J. H. Shin, S.-Y. Seo, K. Kim, and S. G. Bishop, "Photoluminescence excitation spectroscopy of erbium-doped siliconrich silicon oxide," Applied Physics Letters, vol. 76, no. 15, pp. 1999-2001, 2000.

[9] D. Comedi, O. H. Y. Zalloum, E. A. Irving, et al., "X-raydiffraction study of crystalline Si nanocluster formation in annealed silicon-rich silicon oxides," Journal of Applied Physics, vol. 99, no. 2, Article ID 023518, 8 pages, 2006.

[10] C. L. Heng, O. H. Y. Zalloum, J. Wojcik, T. Roschuk, and P. Mascher, "On the effects of double-step anneal treatments on light emission from Er-doped Si-rich silicon oxide," Journal of Applied Physics, vol. 103, no. 2, Article ID 024309, 2008.

[11] J. Li, O. H. Y. Zalloum, T. Roschuk, C. L. Heng, J. Wojcik, and P. Mascher, "Light emission from rare-earth doped silicon nanostructures," Advances in Optical Technologies, vol. 2008, Article ID 295601, 10 pages, 2008.

[12] A. Podhorodecki, J. Misiewicz, J. Wojcik, E. Irving, and P. Mascher, "1.54 $\mu \mathrm{m}$ room temperature emission from Erdoped Si nanocrystals deposited by ECR-PECVD," Journal of Luminescence, vol. 121, no. 2, pp. 230-232, 2006.

[13] F. Iacona, G. Franzò, and C. Spinella, "Correlation between luminescence and structural properties of Si nanocrystals," Journal of Applied Physics, vol. 87, no. 3, pp. 1295-1303, 2000.

[14] G. Ledoux, J. Gong, F. Huisken, O. Guillois, and C. Reynaud, "Photoluminescence of size-separated silicon nanocrystals: confirmation of quantum confinement," Applied Physics Letters, vol. 80, no. 25, p. 4834, 2002.

[15] C. Meier, A. Gondorf, S. Lüttjohann, A. Lorke, and H. Wiggers, "Silicon nanoparticles: absorption, emission, and the nature of the electronic bandgap," Journal of Applied Physics, vol. 101, no. 10, Article ID 103112, 8 pages, 2007.

[16] D. Kovalev, J. Diener, H. Heckler, G. Polisski, N. Künzner, and F. Koch, "Optical absorption cross sections of Si nanocrystals," Physical Review B, vol. 61, no. 7, pp. 4485-4487, 2000. 
[17] G. Allan, C. Delerue, and M. Lannoo, "Electronic structure of amorphous silicon nanoclusters," Physical Review Letters, vol. 78, no. 16, pp. 3161-3164, 1997.

[18] A. Podhorodecki, J. Misiewicz, F. Gourbilleau, and R. Rizk, "Absorption mechanisms of silicon nanocrystals in cosputtered silicon-rich-silicon oxide films," Electrochemical and Solid-State Letters, vol. 11, no. 3, pp. K31-K33, 2008.

[19] M. V. Wolkin, J. Jorne, P. M. Fauchet, G. Allan, and C. Delerue, "Electronic states and luminescence in porous silicon quantum dots: the role of oxygen," Physical Review Letters, vol. 82, no. 1, pp. 197-200, 1999.

[20] Y. M. Niquet, C. Delerue, G. Allan, and M. Lannoo, "Method for tight-binding parametrization: application to silicon nanostructures," Physical Review B, vol. 62, no. 8, pp. 5109-5116, 2000.

[21] A. Podhorodecki, G. Zatryb, J. Misiewicz, J. Wojcik, and P. Mascher, "Influence of the annealing temperature and silicon concentration on the absorption and emission properties of $\mathrm{Si}$ nanocrystals," Journal of Applied Physics, vol. 102, no. 4, Article ID 043104, 2007. 

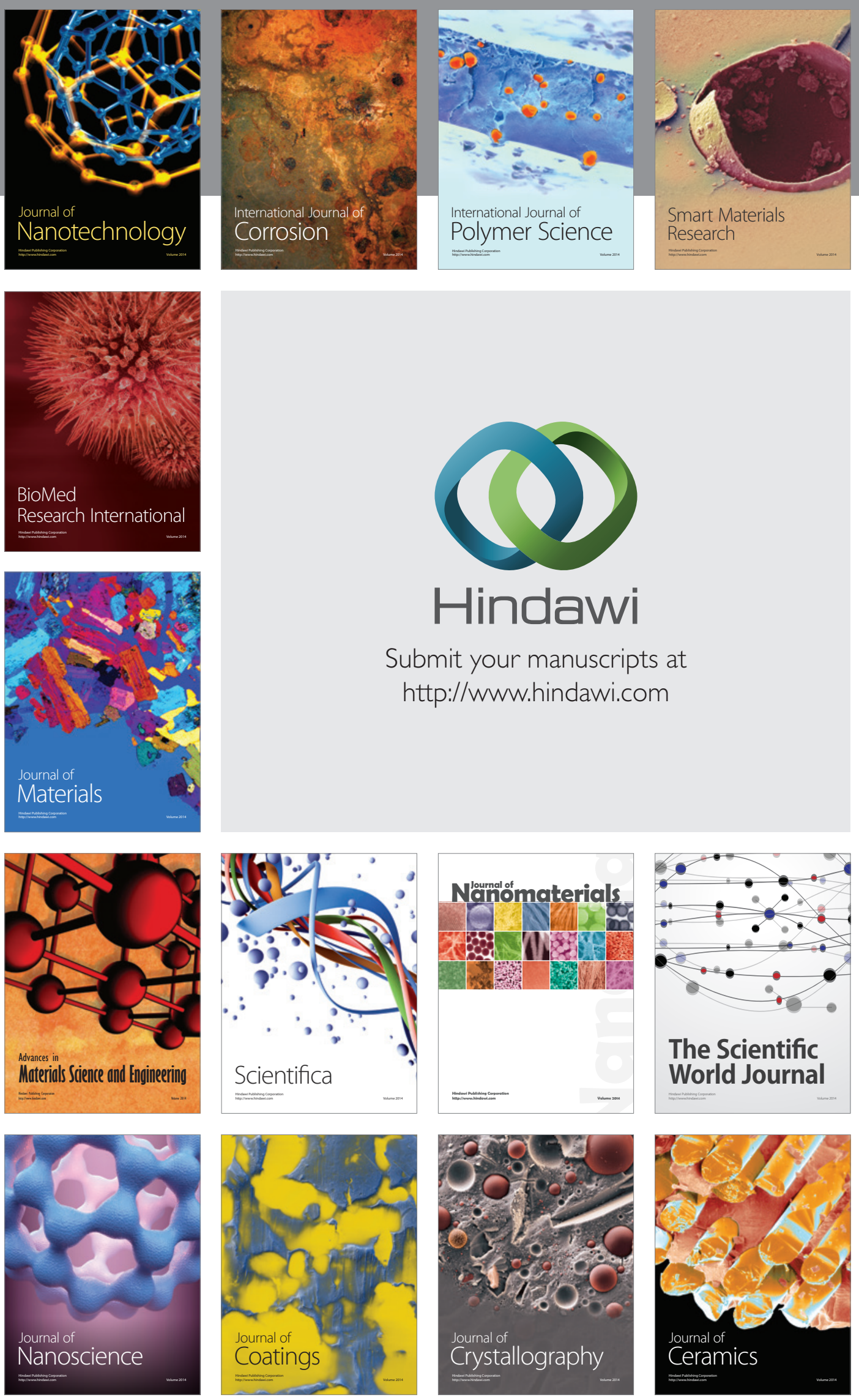

The Scientific World Journal

Submit your manuscripts at

http://www.hindawi.com

\section{World Journal}

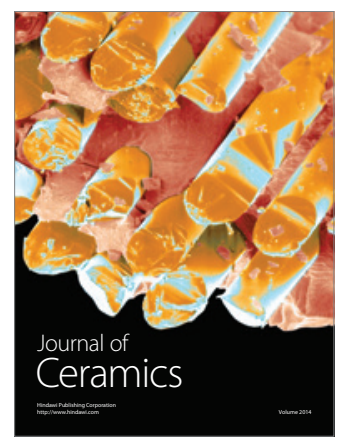

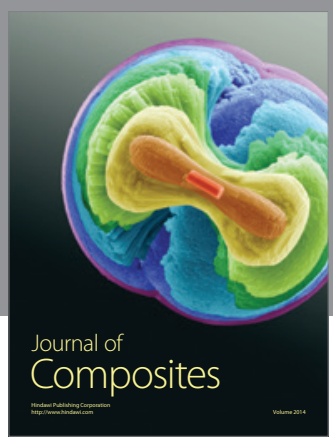
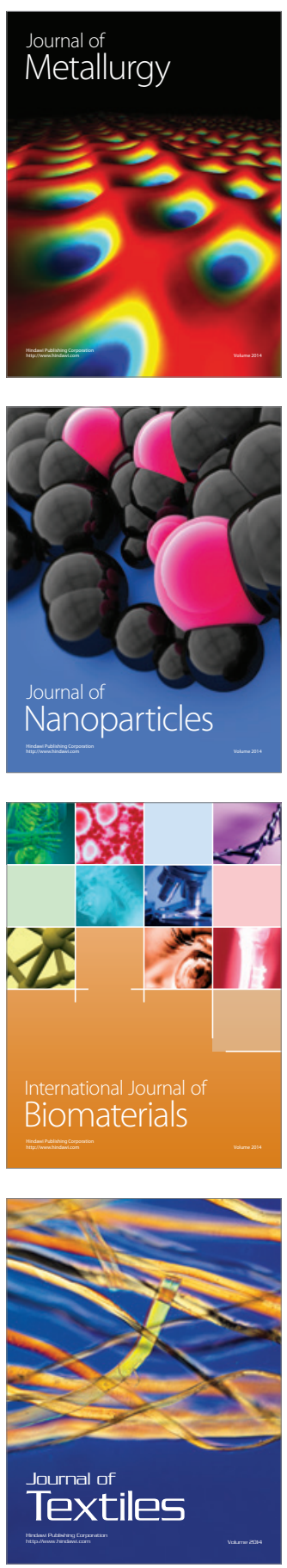